\title{
Broadband metasurfaces for anomalous transmission and spectrum splitting at visible frequencies
}

\author{
Zhongyang Li and Koray Aydin* \\ Department of Electrical Engineering and Computer Science, Northwestern University, 60208 Evanston, IL, US
}

Received 1 September 2015 / Accepted 15 October 2015

\begin{abstract}
The emergent ultrathin metasurfaces are promising optical materials to enable novel photonic functionality and miniature optical devices. By elaborately design the interfacial phase shift from discrete nanoantennas with distinctive geometries, metasurfaces have the potential to shape desired wavefronts and arbitrary steer light propagation. However, the realization of broadband transmission-mode metasurfaces that operates at visible frequencies have still been significant challenging. Because it is difficult to achieve drastic broadband optical response depending on discrete plasmonic resonators and the fabrication of such subwavelength-size resonators with high uniformity is also challenging. Here, we propose an efficient yet a simple transmission-mode metasurface design comprising of a single, quasi-continuous nanoantenna as the build block. Each nanoantenna consist of a trapezoid-shaped triple-layered $\left(\mathrm{Ag}-\mathrm{SiO}_{2}-\mathrm{Ag}\right)$ plasmonic resonator which could induce drastic gradient phase shifts for transmitted light. We numerically demonstrated broadband $(500-850 \mathrm{~nm})$ anomalous transmitted propagation and spectrum splitting at visible frequencies and beyond. The average power ratio of anomalous transmission mode to the first-order diffraction mode was calculated to be $\sim 1000$. Such proposed metasurface design is a clear departure from conventional metasurfaces utilizing multiple discrete resonators, and suggests applications for achieving ultrathin lenses, high SNR spectrometers, directional emitters and spectrum splitting surfaces for photovoltaics.
\end{abstract}

Key words: Metamaterials, Plasmonics, Gradient metasurface, Broadband, Anomalous transmission and reflection, Surface plasmon.

\section{Introduction}

Conventional optical devices including prisms, lenses, mirrors and gratings could control light propagation and shape optical wavefronts either by geometrical optics (propagation, reflection and refraction) or by diffractive optics (diffraction and interferences). However, it is quite challenging to achieve such functionalities when it comes to the regime of nanophotonics. That is because it requires the feature size of optical devices to be larger or at least close to the light wavelength of interest. In addition, the optical property of materials is determined by nature and could not be artificially manipulated. Recently, two-dimensional (2D) metasurfaces, metamaterials of reduced dimensionality, has been emergent and demonstrated to be able to engineer phase retardation along a medium's surface, which opened up new opportunities towards achieving virtually flat optical components at nanoscale [1].

A typical metasurface design usually contains a series of discrete plasmonic nanoantennas with distinctive shape or

*e-mail: aydin@northwestern.edu geometry (size, rotation, density) [2, 3]. Each discrete nanoantenna correspond to a specific resonance phase retardation or amplitude tuning [4]. Through an elaborate arrangement of array along the interface, metasurfaces could realize arbitrary interfacial phase shift and ultimately any desired wavefront shape. So far, the metasurfaces have been demonstrated to enable anomalous reflection and refraction $[5,6]$, beam splitting, beam focusing [7] and other wavefront shaping functionalities [8-10] which could operate at visible, infrared, terahertz, and microwave frequencies. However, it is still quite challenging to achieve visible-frequency metasurfaces with broadband feature to steer transmitted light propagation [11]. It is because the discrete nanoantenna resonance would impart phase shift at narrow-bandwidth spectral region. Therefore, such metasurfaces could only provide limited operation bandwidth and suffer from strong optical losses to the transmitted light due to plasmonic resonances. Here we propose and numerically demonstrate a broadband anomalous meta-transmitarray across the visible frequency range and beyond by utilizing metasurface building block of single gradient "meta-molecule" design. Metasurface design here is a clear departure from conventional 
metasurfaces based on multiple discrete "meta-atom" antenna, and has suggested significant potential for enabling practical applications of flat, ultrathin metasurfaces at visible frequencies and beyond.

\section{Metasurface architecture design}

The designed meta-transmitarray is schematically illustrated in Figure 1. In contrast to the previous building block design containing multiple discrete resonators (meta-atoms), the proposed unit cell here is merely composed of single trapezoid-shaped triple-layered $\left(\mathrm{Ag}-\mathrm{SiO}_{2}-\mathrm{Ag}\right)$ resonator (metamolecule) patterned on glass substrate $(n=1.45)$. Such sample could be fabricated starting with patterning on silica substrate by electron-beam lithography and then following by a sequential deposition of $\mathrm{Ag}$ (30 nm thick), $\mathrm{SiO}_{2}$ (40 nm thick) and $\mathrm{Ag}(30 \mathrm{~nm}$ thick) and finally lifting-off triple-layer structures together. The width size of the trapezoid nanoantenna gradually changes from $40 \mathrm{~nm}$ to $150 \mathrm{~nm}$. The illumination source is normally incident to the metasurface (along the $z$-axis) with polarization (electric-field direction) along $x$-axis, as shown in Figure 1.

Full-field electromagnetic wave calculations were performed using Lumerical ${ }^{\mathrm{TM}}$, a commercially available finitedifference time-domain (FDTD) simulation software package. A unit cell are of $200 \times 1000 \mathrm{~nm}^{2}$ has been utilized for 3D simulation with periodic boundary conditions along $x$ and $y$ axis. Perfectly matched layers (PML) conditions are utilized along the light propagation (z-axis). Broadband plane wave was normally incident to the metasurface along the $z$-axis. The transmitted powers have been collected using a power monitor at far-field. The transmitted powers at a full range of angles are calculated by the far-field calculation option of the transmission power monitor. The complex refractive index of $\mathrm{Ag}$ for simulation is utilized from the data of Palik $(0-2 \mu \mathrm{m})$ and $\mathrm{SiO}_{2}$ is from the data of Palik.

\section{Broadband phase shift}

To understand the interfacial phase shift along trapezoidshaped nanoantenna, one could differentiate the gradient width of trapezoid-shaped antenna into several individual nanorods with uniform width. For a single layer of trapezoid-shaped Ag antenna, the corresponding phase shift has been studied and demonstrated to enable broadband anomalous reflection at visible frequencies [12]. For the transmission mode of broadband metasurface, here each nanorod contains a triple-layer of $\mathrm{Ag}-\mathrm{SiO}_{2}-\mathrm{Ag}$ in order to enhance drastic phase modulation for the transmitted light. When excited by light polarization orthogonal to each nanorod, the interfacial phase shift is continuously tuning when the nanorod changes its width from 40 to $150 \mathrm{~nm}$, exhibiting drastic phase coverage with broadband feature, as shown in Figure 2a. The gradient of the contour curves indicates the phase gradients at different wavelengths. Figures $2 b-2 c$ plot the phase shift for different nanorod width at respective wavelengths of $750 \mathrm{~nm}$ (Figure 2b), $650 \mathrm{~nm}$ (Figure 2c) and $550 \mathrm{~nm}$ (Figure 2d).

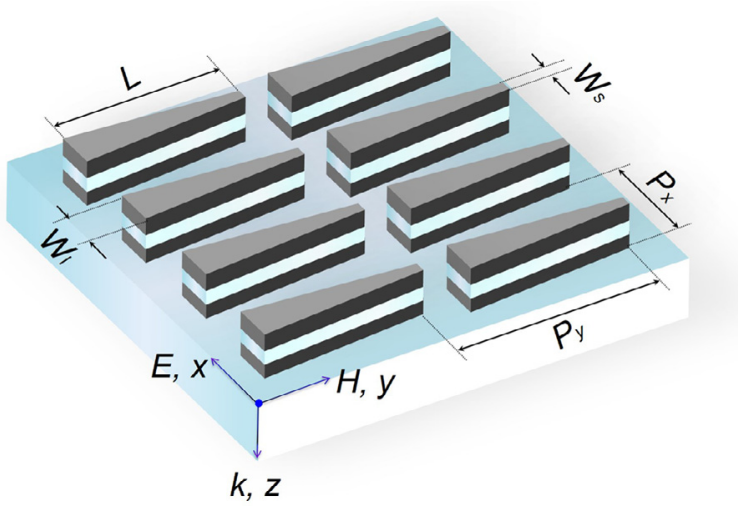

Figure 1. Schematic drawing of trapezoid-shaped triple-layer (Ag$\mathrm{SiO}_{2}-\mathrm{Ag}$ ) nanorod array on glass substrate with geometric parameters of $P_{x}=200 \mathrm{~nm}, P_{y}=1000 \mathrm{~nm}, W_{s}=40 \mathrm{~nm}, W_{l}=150 \mathrm{~nm}$ and $L=800 \mathrm{~nm}$. The triple-layer nanorod thickness is $30 \mathrm{~nm}(\mathrm{Ag})$, $40 \mathrm{~nm}\left(\mathrm{SiO}_{2}\right)$ and $30 \mathrm{~nm}(\mathrm{Ag})$, respectively. Broadband white source is normally (along $z$-axis) incident onto the sample surface with the electric-field polarized along $x$-axis.

Specifically, the phase shift gradient is relatively flat at $550 \mathrm{~nm}$ and steeper at $750 \mathrm{~nm}$. By merging such individual uniform nanorods into a simple trapezoid-shaped nanoantenna, the phase shift could be continuously modulated along the metasurface by changing its width from $W_{s}=30 \mathrm{~nm}$ to $W_{l}=150 \mathrm{~nm}$. Overall, the interfacial phase shift induced by metasurface could cover from 500 to above $800 \mathrm{~nm}$ in visible frequencies.

Such induced abrupt phase discontinuity would provide an effective interfacial wavevector and thus steer the "normal" transmitted beam to an off-normal direction determined by the gradient of the phase change. According to the generalized Snell's Law, the transmission angle of outgoing beam is reduced to

$$
\theta_{t}=\arcsin \left(\frac{\lambda_{o}}{2 \pi} \frac{d \Phi}{d y}\right)
$$

Additionally, due to the wavelength dispersion and the wavelength-dependent phase gradients $\left(\frac{d \Phi}{d y}\right)$, different frequency components of the visible light would be steered to different transmission angles. Therefore, the outgoing beam would be featured with rainbow colors, which is similar to the phenomenon of reflection-mode of metasurfaces.

\section{Broadband anomalous transmission beam}

Figure 3a shows the simulated all-directional transmission spectra as a function of wavelength and the transmission angle at far-field from the metasurface. There are basically five diffraction modes exhibiting on the transmission spectra, i.e., the normal transmission mode $(m=0)$, the first-order and the second-order anomalous transmission modes $(m=1,2)$ at the positive-angle side and the first-order and second-order diffraction mode $(m=-1,-2)$ at the negative-angle side. It is worth noting that in addition to normal propagation light, stronger transmitted light power is located at the first-order 

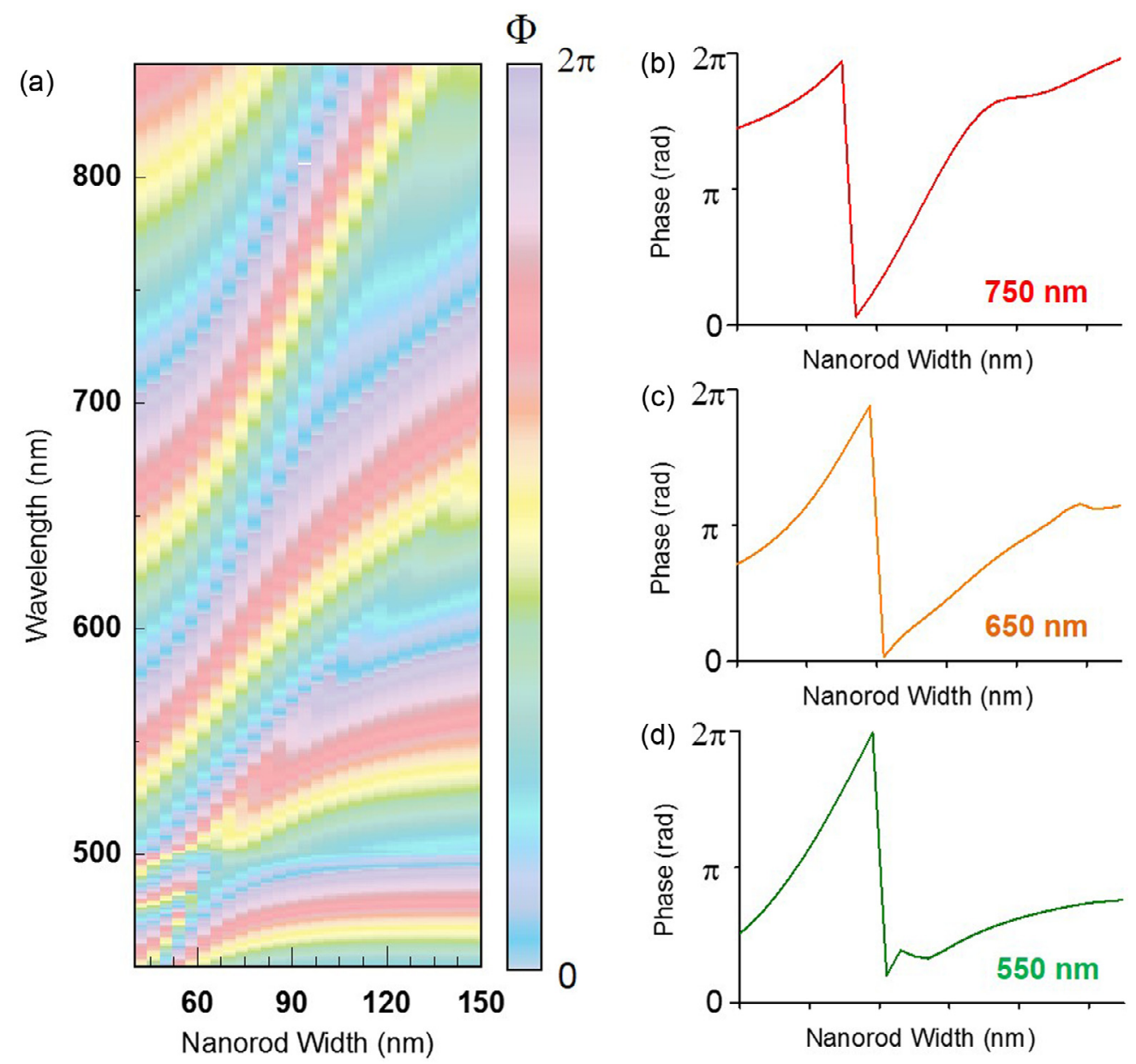

Figure 2. (a) Simulated 2D contour for phase shifts of nanorods as a function of width size and wavelength. (b-d) Simulated phase shift plots as a function of nanorod width at wavelength of $750 \mathrm{~nm}, 650 \mathrm{~nm}$ and $550 \mathrm{~nm}$, respectively.
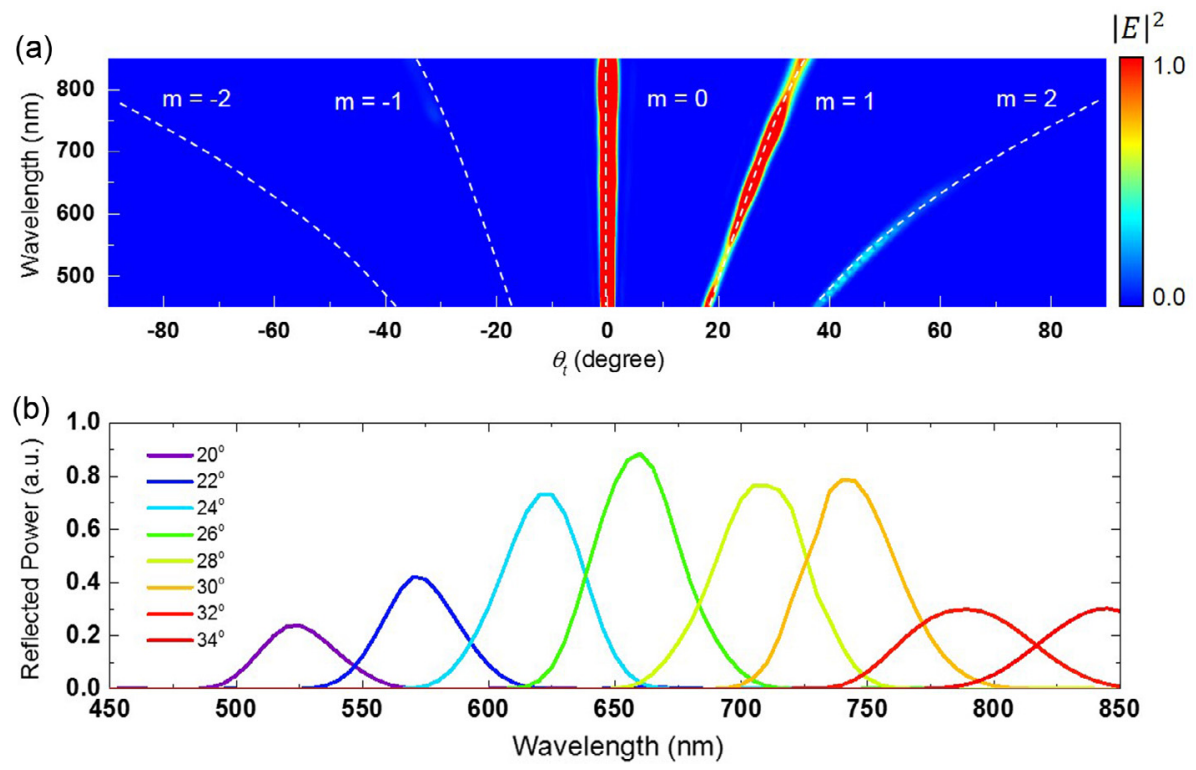

Figure 3. Angular distribution of the simulated transmitted power at far-field from the metasurface. (a) Simulated $2 \mathrm{D}$ contour for far-field transmitted powers as a function of transmission angles $\theta_{t}$ (x-axis) and wavelength ( $y$-axis). (b) Simulated anomalous transmitted power spectra for different transmission angles from $20^{\circ}$ to $34^{\circ}$ with $2^{\circ}$ interval, respectively. 

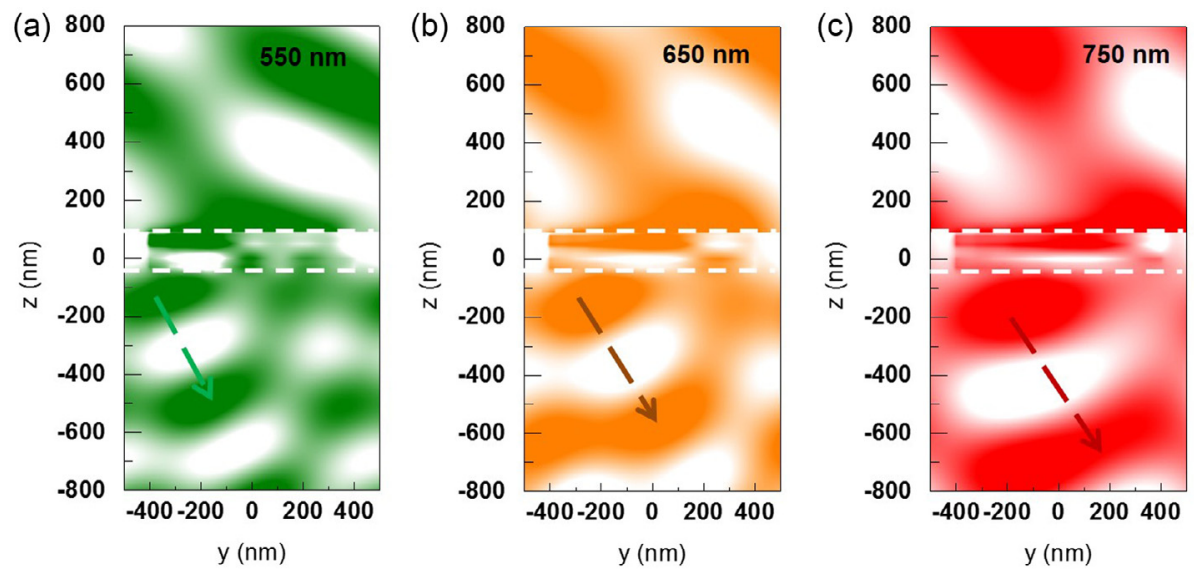

Figure 4. Calculated near-field profiles and wavefront shapes of the transmitted waves below the triple-layered $\left(\mathrm{Ag}-\mathrm{SiO} \mathrm{O}_{2}-\mathrm{Ag}\right) \mathrm{resonator}$ arrays at $550 \mathrm{~nm}, 650 \mathrm{~nm}$ and $750 \mathrm{~nm}$, respectively. The directions of the arrows denote the propagation direction of transmitted waves and the lengths of the arrows indicate the wavelength size of each frequency component of light.

anomalous transmission mode at oblique transmission angles from $\sim 20^{\circ}$ to $\sim 40^{\circ}$. Very minor transmitted power is scattered to the first diffraction order to the negative-angle side. Therefore, except the normal transmitted propagation light, most of the outgoing photons are redirected to propagate along oblique transmission angles. Moreover, such anomalous transmitted light contains broadband frequency components, covering visible spectra from below $500 \mathrm{~nm}$ to above $800 \mathrm{~nm}$. For the second-order diffractions, the light power located at the positive-angle mode $(m=2)$ is also much stronger than the negative-angle mode $(m=-2)$. Figure $3 b$ plots the simulated transmission spectra for the first-order anomalous mode, respectively, scanning at detection angles between $\sim 20^{\circ}$ and $\sim 40^{\circ}$ with $2^{\circ}$ interval. The bandwidth of the simulated transmitted power spectra at a specific angle is $\sim 50 \mathrm{~nm}$, indicating low angular dispersion for the transmitted light. Comparing the power intensities between $m=1$ and $m=-1$ modes, the average power ratio of anomalous transmitted mode $(m=1)$ to the first diffraction order $(m=-1)$ is calculated to be on the order of $10^{3}$. The maximum conversion efficiency from the incoming light to anomalous outgoing light occurs around $600-750 \mathrm{~nm}$, where the highest power ratio between $m=1$ and $m=-1$ modes takes place.

To better illustrate the anomalous propagation of these outgoing photons from metasurface, the cross-section electricfield profiles were calculated to demonstrate the modulated wavefronts shape of transmitted light. The cross-section electric field distributions are detected by a 2D field monitor placed at $y-z$ plane $(x=0)$. The electric profile range is $1000 \mathrm{~nm}$ along $y$-axis, covering the entire building block and $1600 \mathrm{~nm}$ along $z$-axis. Figures $4 \mathrm{a}-4 \mathrm{c}$ show the calculated electric-field profiles of the transmitted wavefronts at specific wavelengths of $550 \mathrm{~nm}, 650 \mathrm{~nm}$ and $750 \mathrm{~nm}$, respectively. The transmission angles are slightly different for different frequency components of light due to the phase gradients dispersion. Specifically, the transmitted light at $550 \mathrm{~nm}$ would propagate along an oblique angle of $\sim 21^{\circ}$ and for the wavelength of
$650 \mathrm{~nm}$ and $750 \mathrm{~nm}$, the transmission angles are $\sim 25^{\circ}$ and $\sim 28^{\circ}$, respectively. It is observed that the wavefront shape of transmitted light exhibits strong interference patterns below the metasurface structures, which is due to a strong interference effect between the anomalous transmitted light and the normal transmitted light.

Furthermore, it is worth noting that such anomalous transmission based on metasurfaces is conceptually different from the conventional blazed gratings with triangular, sawtoothshaped grooves. By varying the groove depth, blazed gratings accumulate gradual linear phase delay through different length of light propagation path, essentially similar with conventionally oblique refraction from an inclined plane. However, the proposed metasurface arrays enable the linear/non-linear phase discontinuities along a flat 2D surface, fundamentally based on excitation localized plasmonic modes from anisotropic resonators with subwavelength-size perpendicular to the incidence plane.

\section{Conclusion}

In summary, we have numerically proposed a transmissionmode metasurface design by quasi-continuous trapezoidshaped nanoantenna arrays and demonstrated broadband anomalous transmission metasurfaces at visible frequencies. The designed building block consists of a triple-layered (Ag$\mathrm{SiO}_{2}-\mathrm{Ag}$ ) plasmonic resonator to induce drastic phase shift for the transmission light. Through the gradually modulated phase shift along the interface, the metasurface could enable broadband anomalous transmission and spectrum splitting functionalities. Such virtually flat metasurfaces suggest new possibilities for integration into other photonic and plasmonic devices [13] and are promised to impact wide variety of applications such as ultrathin lenses [14, 15], high SNR optical spectrometer, polarization beam splitters, high efficiency plasmon couplers, directional emitters, and spectrum splitters for photovoltaics. 


\section{Conflict of interest}

The authors have no competing financial interests.

Acknowledgements. This material is based upon work supported by the AFOSR under Award No. FA9550-12-1-0280. K.A. acknowledges financial support from the McCormick School of Engineering and Applied Sciences at Northwestern University and partial support from the Institute for Sustainability and Energy at Northwestern (ISEN) through ISEN Equipment and Booster Awards. This research was also partially supported by the Materials Research Science and Engineering Center (NSF-MRSEC) (DMR-1121262) of Northwestern University. Z.L. gratefully acknowledges support from the Ryan Fellowship and the Northwestern University International Institute for Nanotechnology.

\section{References}

1. N. Yu, F. Capasso, Nat. Mater. 13 (2014) 139.

2. S. Sun, K.-Y. Yang, C.-M. Wang, T.-K. Juan, W.T. Chen, C.Y. Liao, Q. He, S. Xiao, W.-T. Kung, G.-Y. Guo, L. Zhou, D.P. Tsai, Nano Lett. 12 (2012) 6223.

3. N. Yu, P. Genevet, M.A. Kats, F. Aieta, J.-P. Tetienne, F. Capasso, Z. Gaburro, Science 334 (2011) 333.

4. G. Li, S. Chen, N. Pholchai, B. Reineke, P.W.H. Wong, E.Y.B. Pun, K.W. Cheah, T. Zentgraf, S. Zhang, Nat. Mater. 14 (2015) 607.
5. X. Ni, N.K. Emani, A.V. Kildishev, A. Boltasseva, V.M. Shalaev, Science 335 (2012) 427.

6. X. Zhang, Z. Tian, W. Yue, J. Gu, S. Zhang, J. Han, W. Zhang, Adv. Mater. 25 (2013) 4566.

7. A. Pors, M.G. Nielsen, R.L. Eriksen, S.I. Bozhevolnyi, Nano Lett. 13 (2013) 829.

8. E.T.F. Rogers, J. Lindberg, T. Roy, S. Savo, J.E. Chad, M.R. Dennis, N.I. Zheludev, Nat. Mater. 11 (2012) 432.

9. S. Sun, Q. He, S. Xiao, Q. Xu, X. Li, L. Zhou, Nat. Mater. 11 (2012) 426.

10. G. Zheng, H. Mühlenbernd, M. Kenney, G. Li, T. Zentgraf, S. Zhang, Nature Nanotechnol. 10 (2015) 308.

11. C. Argyropoulos, K.Q. Le, N. Mattiucci, G. D'Aguanno, A. Alu, Phys. Rev. B 87 (2013) 205112.

12. Z. Li, E. Palacios, S. Butun, K. Aydin, Nano Lett. 15 (2015) 1615.

13. A.A. High, R.C. Devlin, A. Dibos, M. Polking, D.S. Wild, J. Perczel, N.P. de Leon, M.D. Lukin, H. Park, Nature 522 (2015) 192.

14. F. Aieta, P. Genevet, M.A. Kats, N. Yu, R. Blanchard, Z. Gaburro, F. Capasso, Nano Lett. 12 (2012) 4932.

15. F. Aieta, M.A. Kats, P. Genevet, F. Capasso, Science 347 (2015) 1342.

Cite this article as: $\mathrm{Li} \mathrm{Z} \&$ Aydin $\mathrm{K}$ : Broadband metasurfaces for anomalous transmission and spectrum splitting at visible frequencies. EPJ Appl. Metamat. 2015, 2, 2. 